\title{
Channel Estimation for Mimo-Ofdm Systems of Joint Angle-of-Departure (AOD) or Angle-of- Arrival (AOA)
}

\author{
S. Kiranmayi, K. Krishna Murthy
}

\begin{abstract}
Beamforming with gathering mechanical assembly displays has been considered as an engaging advancement in future remote correspondence structures. To coordinate beamforming, one has to know the angle-of-departure (AoD) or angle-of-arrival (AoA). For data disclosure, the beneficiary in like manner has to know station response. In this paper, we propose another joint $\mathrm{AoD}, \mathrm{AoA}$, and channel estimation contrive for pilot-helped MIMO-OFDM systems. Starting, a compressive distinguishing system is used to survey the channel drive response, abusing the sparsity property of remote channels. By then, AoA and AoD are as one surveyed for each perceived path by the most maximum likelihood $(M L)$ procedure. The successive Cramer-Rao lower bound (SCRLB) is moreover gathered and a transmit beamforming plan is proposed as necessities be. In the circumstance of available prior information, a most outrageous a posteriori estimation is proposed. Amusement results exhibit that the proposed procedures can approach the SCRLB achieve a comparable supernatural viability as that got with the ideal direct in millimetre-wave correspondences. the SCRLB is low multifaceted nature computation and to constrain misstep rate and improve sign to fuss extent.
\end{abstract}

Index Terms-Digital communication, maximum likelihood estimation, millimetre wave communication, MIMO systems, multipath channels

\section{INTRODUCTION}

Purpose of-passage (AoA) and the edge of-flight (AoD) is radar, sonar, restriction, and remote exchanges [1]-[3]. In remote trades, it is striking that beamforming can give better banner quality, reduce hindrance, and improve structure limit. Regardless, to coordinate beamforming, we need to know AoA (for get beamforming) or AoD (for transmit beamforming). Starting late, the general media transmission affiliation third time association adventure (3GPP) has begun the regulation method for the bleeding edge system, known as $5 \mathrm{G}$, in which millimeter-wave (mmWave) correspondence [4], [5] plays a key advancement. Because of the extraordinary pathloss in mmWave spread, beamforming with tremendous scale recieving wire bunches transforms into an undeniable prerequisite [6], [7] [8] [9]. Well ordered guidelines to lead capable AoA and AoD estimation is a critical and essential issue. Different sign course of action (MUSIC) and estimationof-signalparameters-by methods for rotational-invariance-procedures (ES PRIT) [10] are two comprehended subspace-based AoA

Revised Manuscript Received on September 10, 2019. college, Movva, A.P, India

K. Krishna Murthy. (Retd) Department of Electronics P.B Siddhartha college of arts and Science, Vijayawada. Presently working at NRI Institute of technology, Agiripalli, A.P, India. extensively utilized in various applications, for instance,

S. Kiranmayi, Department of Electronics V.S.R. Govt. degree \& P.G

estimation strategies. Note that these techniques are outwardly debilitated, suggesting that no planning data are required. In [11]-[13], the subspacebased system is extended to direct joint AoD, AoA and concede estimation. In any case, channel-inspiration responses (CIR) from all transmit radio wires to all get recieving wires are evaluated. By then, the evaluations are organized to shape an estimation vector, and the relationship matrix of the vector is resolved. It will in general be shown that each reason vector in the related subspace joins the transmit bunch response vector, the get display response vector, and the deferrals of the channel ways. Finally, the subspace-based technique is associated with survey the AoDs, AoAs and the way delays. The procedure requires a high computational unusualness. Allow NT to be the amount of transmit gathering mechanical assemblies, NR be the amount of get recieving wires, and $\mathrm{L}$ be the seen as channel length. The computational complexity of the system is O(N3 T N3 RL 3 ), which is denied in the structures with huge recieving wires. Note that this procedure isn't outwardly debilitated any more extended since the CIRs must be assessed first. While it needs many getting ready pictures to assess all the NT NR CIRs, extra planning pictures are moreover required to finish subspace-based techniques, achieving another colossal overhead issue. As opposed to using the subspacebased method, the works in [7], [14] abuse the sparsity of expansion channels and propose a joint AoD, AoA, and channel estimation plot for mmWave correspondences. In mmWave circumstances, the amount of ways is regularly compelled and the CIR is thusly pondered lacking in time and exact territories [15], [16]. The methodology quantizes the AoA and AoD into explicit levels and treats the edge estimation as a recognizable proof issue. In [7], a close-by circle shaft getting ready is proposed. At each stage, the transmitter beamforms the transmitted sign in one part of AoDs and the recipient beamforms the get signal in one parcel of AoAs. At the completion of each stage, the best AoD and AoA are picked as the reference for the accompanying stage. The whole framework is repeated and the beamforming is proposed for each phase in like way. The obstruction of the methodology is that the planning time can be long if better dapper precision is required. In [14], an open-circle bar planning is proposed. The beamforming is organized by the soundness property in the compressiveidentifying theory to such a degree, that the required planning time can be diminished. Note that the two

\section{Published By:}

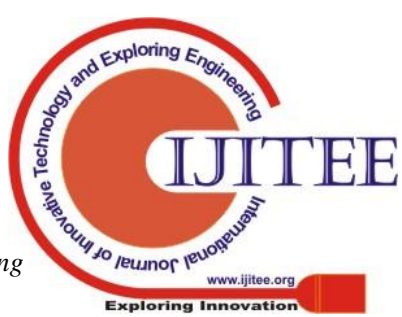


strategies discussed above simply consider the narrowband channel. Furthermore, the assessed focuses are quantized. In this paper, we consider the joint AoD, AoA and direct estimation in pilot-helped MIMO-OFDM structures.

The wideband channel is considered i.e., the CIR involves unmistakable multi-ways. As the standard supposition in most composition for channel estimation, we acknowledge that each scatterer contributes one single route parameterized by its deferral, expansion, AoD and AoA. In our OFDM systems, pilot sign is installed in certain subcarries for channel estimation, and various planning pictures are transmitted with different beamforming vectors. After CIR estimation, all deferments and increments of the channel ways would then have the option to be gotten from which AoD and AoA can be evaluated as necessities be. It is understood that remote channels are as often as possible meager and the CIR estimation can be seen as a compressive-distinguishing (CS) issue [17]. In like manner, the CS issue can be understood by some low-multifaceted design method, for instance, organizing interest (MP). The customary CS-based CIR estimation strategy just considers the single get gathering mechanical assembly circumstance. In this work, we at first propose a redesigned MPbased estimation taking the diverse get recieving wires and different beamforming transmissions into idea. At the point when the CIR is assessed, the best likelihood (ML) procedure is used to evaluate AoD and AoA. As will be showed up, there exists a covered parameter, i.e, the route gain, in the likelihood work. Thusly, the longing intensification (EM) figuring [18] [19] is associated. We decide the looking at Cram'er-Rao lower bound (CRLB) and propose a transmit beamforming plan, upgrading a CRLBrelated limit, moreover. One of our past works [20] just considers AoA and channel estimation while the other [21] just considers AoD and channel estimation. Here, we stretch out these systems to a joint AoA, AoD and channel estimation.

\section{SYSTEM MODEL}

Consider a MIMO-OFDM system. Allow L to be the amount of routes in the CIR, NT be the amount of recieving wires in the transmitter, and NR be that in the gatherer. Moreover, let $\varphi \mathrm{l}$ be the AoD and 91 be the AoA of the lth way. Acknowledge that the uniform direct display is used at transmitter and authority. By then, the bunch response at the transmitter, implied by aT, $1 \in \mathrm{C}$ NT $\times 1$, can be created by its part $[\mathrm{aT}, 1] \mathrm{m}=\exp (\mathrm{j}(\mathrm{m}-1) \varphi \mathrm{l})$, addressing the stage proceed onward the mth transmit recieving wire. Along these lines, the show response at the recipient, demonstrated by $\mathrm{aR}, 1 \in \mathrm{C} \mathrm{NR} \times 1$, is gotten by $[\mathrm{aR}, 1] \mathrm{m}=\exp (\mathrm{j}(\mathrm{m}-1) \vartheta \mathrm{l})$. Mean $\alpha \mathrm{l} \in \mathrm{C}$ as the route expansion of the lth way. By then, the timeterritory CIR, demonstrated by $\mathrm{C}(\mathrm{n})$, can be formed as:

$$
\mathbf{C}(n)=\sum_{l=1}^{L} \alpha_{l} \mathbf{a}_{R, l} \mathbf{a}_{T, l}^{T} \delta\left(n-k_{l}\right),
$$

where $\mathrm{kl}$ is the concede time of the lth channel way. Without loss of accord, we acknowledge that $\mathrm{k} 1$ $<\mathrm{k} 2<$. $<\mathrm{kL}$. Note that the channel response for a route at a particular minute is a network. For the AoD and AoA estimation, Q OFDM pictures are engineered to transmit. Furthermore, the transmitter practices transmit beamforming for each transmission and the beamformed

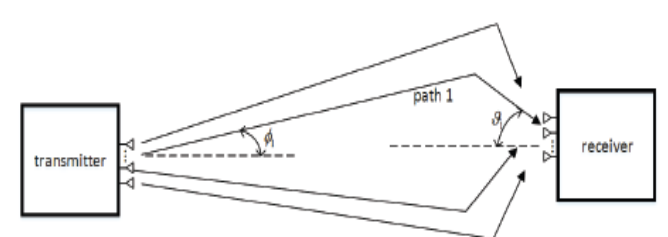

Fig. 1. Illustration of the beamformed channel model in the qth beamforming transmission.

CIR is seen at authority. Show wq $\in$ C NT $\times 1$ as the beamforming vector in the qth beamforming transmission. The beamformed CIR in the qth beamforming transmission, demonstrated by $\mathrm{cq}(\mathrm{n})$, can be formed as:

$$
\mathbf{c}_{q}(n)=\sum_{l=1}^{L} \alpha_{l} \mathbf{a}_{R, l} \mathbf{a}_{T, l}^{T} \mathbf{w}_{q} \delta\left(n-k_{l}\right) .
$$

Fig. 1 illustrates for the beamformed channel model with $\mathrm{L}=4$ in (2). In the OFDM system, the size of the cyclic prefix (CP), demonstrated by $\mathrm{I}$, is believed to be greater than the best defer time $\mathrm{kL}$ [22]. In a pilot-helped OFDM system [23], the pilot pictures are transmitted unmistakably in some specific subcarriers. We at present depict the sign model at the mth get gathering mechanical assembly in the qth beamforming transmission. Allow $\mathrm{N}$ to be the size of the discreteFourier-change (DFT), $\mathrm{P}$ be the amount of transmitted pilots in subcarriers, $\mathrm{P}$ be the record set of the pilot

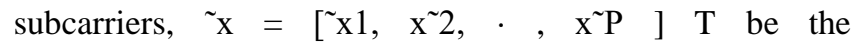
transmitted pilots on the $\mathrm{P}$ subcarriers with documents $\mathrm{P}, \mathrm{I}=\{1,2, \ldots, \mathrm{I}\}$, and $\mathrm{G} \in \mathrm{C} \mathrm{N} \times \mathrm{N}$ be the DFT organize. For the mth got gathering mechanical assembly, the corresponding CIR, implied by hq, $\mathrm{m} \in$ C $\mathrm{I} \times 1$, can be procured from :

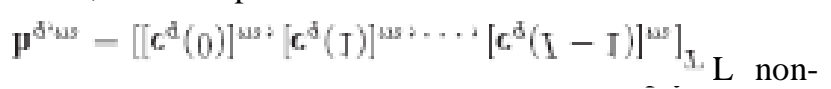

zero segments in hq,m and its lth non-zero worth is al $\exp \left(\mathrm{j}(\mathrm{m}-1)\right.$-1)a $\mathrm{T} \mathrm{T}, \mathrm{lwq}$ at deferment $\mathrm{kl}$. Mean $\mathrm{h}^{\sim}$ $\mathrm{q}, \mathrm{m}$ as the channel repeat response on the $\mathrm{P}$ pilot subcarriers, by then $\mathrm{h}^{\sim} \mathrm{q}, \mathrm{m}=$ Fhq, $\mathrm{m}$, where $\mathrm{F}=$ [G]P,I. It is anything but difficult to see that $[\mathrm{F}] \mathrm{p}, \mathrm{n}=$ $\exp (-j 2 \pi(P(p)-1)(n-1) / N) / \sqrt{ } N$. The got pilots in an OFDM picture, shown by rq, $\mathrm{m}$, can be made by $\sim \mathrm{rq}, \mathrm{m}=\operatorname{diag}(\sim \mathrm{x}) \mathrm{h}^{\sim} \mathrm{q}, \mathrm{m}+\tilde{\mathrm{nq}}, \mathrm{m}$, where $\tilde{\mathrm{nq}, \mathrm{m}}$ is the contrasting bustle vector. Note that the pilot pictures are customarily QPSK balanced with unit control. Along these lines, it might be adequately removed

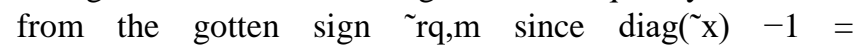
$\operatorname{diag}(\sim x) H$ is only a phase transformation. In the wake of emptying the pilot pictures, the evaluated 
channel repeat response, shown by $h^{2} \mathrm{q}, \mathrm{m}$, can be made by:

$$
\begin{aligned}
\check{\mathbf{h}}_{q, m} & =\tilde{\mathbf{h}}_{q, m}+\check{\mathbf{n}}_{q, m} \\
& =\mathbf{F} \mathbf{h}_{q, m}+\check{\mathbf{n}}_{q, m}
\end{aligned}
$$

where $\mathrm{n} \check{\mathrm{q}}, \mathrm{m}=\operatorname{diag}(\sim \mathrm{x}) \mathrm{Hn}^{\sim} \mathrm{q}, \mathrm{m}$ is the rotated noise vector assumed to be $\mathrm{CN}(0, \sigma 2 \mathrm{nI})$.

Algorithm 1 : Subspace Pursuit Algorithm

Information: 1: $\mathrm{F}$ : inspecting lattice

2: $h^{\checkmark} \mathrm{q}, \mathrm{m}$ : recurrence space watched channel at the mth reception apparatus in the qth beamforming transmission

Yield: CIR estimation $\mathrm{h}^{\wedge} \mathrm{q}, \mathrm{m}$ : with non-zero components being equivalent to $\mathrm{h}^{\wedge} \mathrm{Ri} q, \mathrm{~m}$ at lists $\mathrm{Ri}$

3: Initialization:

4: Conduct the coordinating activity g $0 \mathrm{q}, \mathrm{m}=\mathrm{F}$ $\mathrm{Hh}^{`} \mathrm{q}, \mathrm{m}$

5: $\mathrm{R} 0=\{\mathrm{kl}, 1=1, \ldots, \mathrm{Ls}\}$ : lists with the Ls biggest $\ell 1$-standard $\mathrm{d} 1(\mathrm{l})=\mid \mathrm{PNR} \mathrm{m}=1$ PQ $\mathrm{q}=1\left[\begin{array}{ll}\mathrm{g} & 0\end{array}\right.$ $\mathrm{q}, \mathrm{m}] \mathrm{l}$ |

6: s 0 q,m $=(\mathrm{I}-[\mathrm{F}]:, \mathrm{R} 0[\mathrm{~F}] \dagger$ :, R0 $) \mathrm{h}^{2} \mathrm{q}, \mathrm{m}$

7: rehash

8: At the ith emphasis

9: Conduct the coordinating activity $\mathrm{g} \mathrm{I} \mathrm{q}, \mathrm{m}=\mathrm{F} \mathrm{Hs}$ i-1 q,m 10: $\mathrm{Ki}$ : files with the Ls biggest $\ell 1$-standard $\mathrm{d} 1(\mathrm{l})=\mid \mathrm{PNR} \mathrm{m}=1$ PQ q=1[g I q,m]l |

11: $\mathrm{S} \mathrm{I}=\mathrm{Ri}-1 \cup \mathrm{Ki}$

12: h^S I q,m $=[F] \dagger:, S^{2}{ }^{`}$ q,m

13: $\mathrm{Ri}:$ lists with the Ls biggest $\ell 1$-standard $\mathrm{d} 2(\mathrm{l})$ $=$ PNR $\mathrm{m}=1$ PQ $\mathrm{q}=1 \mid\left[\mathrm{h}^{\wedge} \mathrm{S}\right.$ I q,m]l $\mid$

14: CIR Estimation $h^{\wedge}$ Ri q,m $=[F] \dagger:$, Rih $^{`} \mathrm{q}, \mathrm{m}$

15: s I q,m $=h^{`}$ q,m - [F]:,Rih^ Ri q,m

16: until stop measure.

\section{SIMULATION RESULTS I}

Here, we report diversion results demonstrating the display of the proposed estimations. In Section VI-A, we survey the introduction of the proposed edge estimation, differentiated and the decided CRLB and BCRLB. In Section VI-B, we inquire about the reachable powerful profitability of the proposed system in mmWave correspondence. For EM count, the amount of emphasess is set as 10. The sign tonoiseratio (SNR) edge $\rho t$ is set as 5 in the Newton's technique (M-step). Exactly when the way SNR is greater than the farthest point, the amount of update is set as 20. Something different, the amount of update is set as 40 with $\kappa=0.1$. For BEM computation, the amount of cycles is set as 10 and the amount of update in the Mstep is set as 20. The stepsize of AoA BEM and AoD BEM estimations are set as 0.5 and 0.05 , independently.

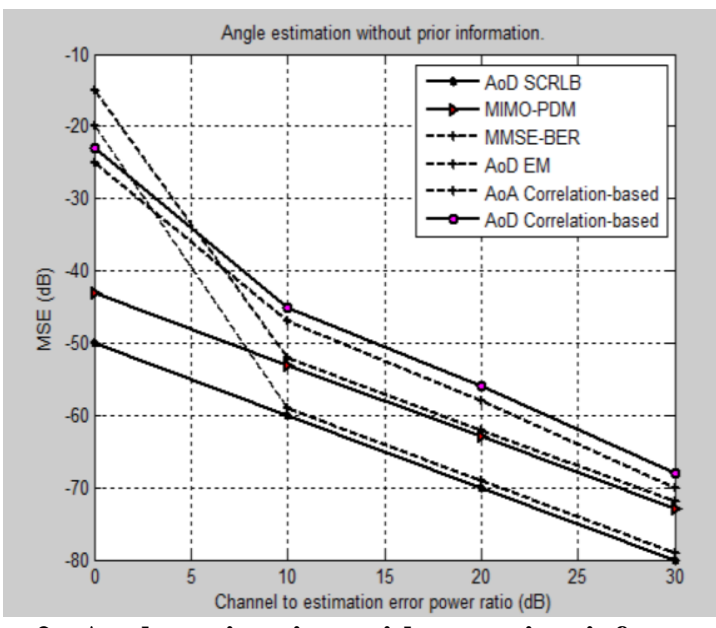

Fig. 2. Angle estimation without prior information

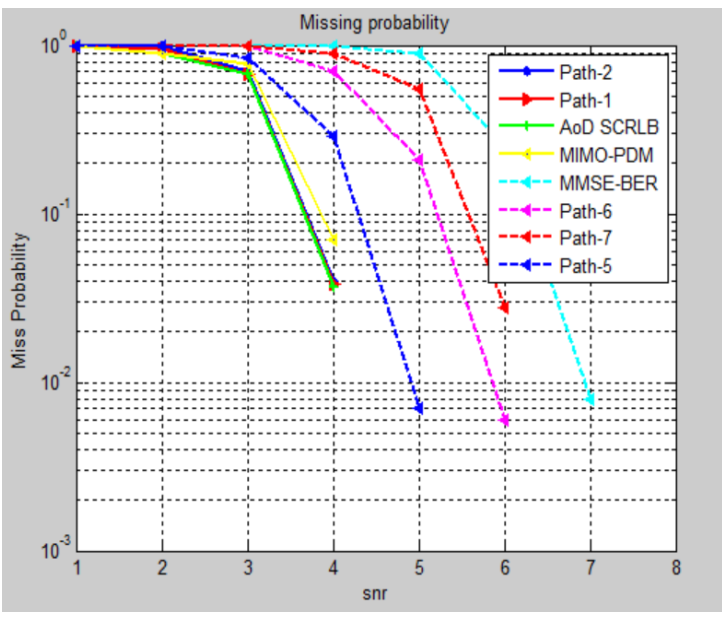

Fig. 3. Missing probability

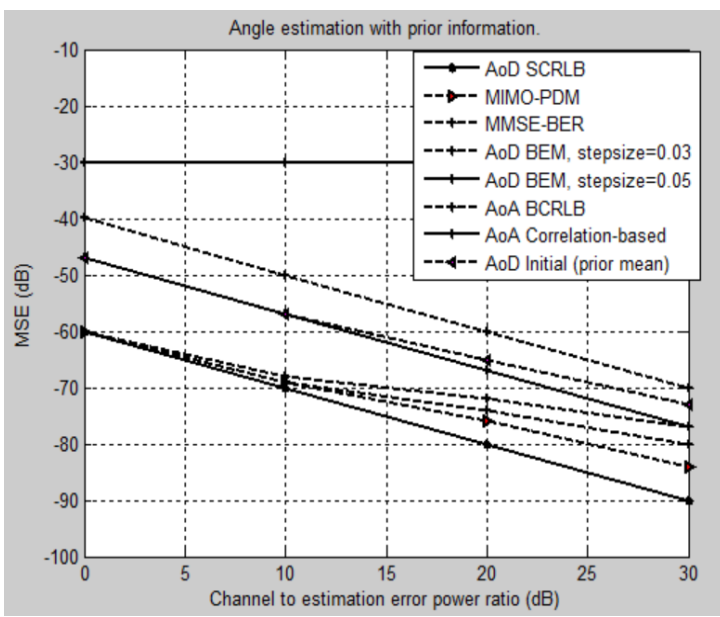

Fig. 4. Angle estimation with prior information

\section{CONCLUSIONS}

In this paper, we consider join AoD, AoA, and channel estimation for pilot-helped OFDM systems. Not exactly equivalent to standard methodologies, the wide-band channel model is considered. Our system decouples AoD/AoA and channel estimations with the ultimate objective that the computational complexity 
can be fantastically lessened. In any case, we propose another MP-based CS based strategy for CIR estimation, abusing the sparsity of the CIR in time zone. With the assessed channels, the AoD and AOA would then have the option to be surveyed for each distinguished way. Two-point estimation methodologies related with two transmit beamforming plans are proposed, contrasting with the case without or with prior information. For the chief case, the CRLB is resolved and the ML estimation, completed with the EM count, is coordinated. The beamforming plan is proposed to propel a CRLB-related limit. Proliferations show that the display of the proposed strategy can accomplish the CRLB. For the consequent case, the BCRLB is resolved and the transmit beamforming, upgrading a BCRLB-related limit, is proposed. Moreover, the MAP estimation with a two-orchestrate BEM figuring is proposed. Reenactments show this proposed technique can accomplish the BCRLB as well. Finally, the proposed methodologies are associated in mmWave correspondences. Amusements exhibit that the proposed techniques can achieve a comparative farthest point as that of the ideal channel, empowering its utilization in future $5 \mathrm{G}$ structures. While the proposed procedures acknowledge the use of cutting edge displays, they can in like manner be extended to the circumstance of other group structures, e.g., blend show. Similarly, each channel way is relied upon to related with a single occupy tap in this paper. When in doubt, each way may join a lot of channel taps. The best strategy to deal with these issues merits further examination. Research in these topics is as of now in advancement.

\section{REFERENCE}

1. L. Xu, J. Li, and P. Stoica, "Target detection and parameter estimation for MIMO radar systems," IEEE Transactions on Aerospace and Electronic Systems, vol. 44, pp. 927-939, July 2008.

2. A. Kangas, I. Siomina, and T. Wigren, Handbook of Position Location: Theory, Practice and Advances, R. Zekavat and R. Buehrer, Eds. 445 Hoes Lane, Piscataway, NJ 08854, USA: Wiley-IEEE Press, 2012.

3. M. Chryssomallis, "Smart antennas," IEEE Antennas and Propagation Magazine, vol. 42, pp. 129-136, June 2000.

4. F. Boccardi, R. W. Heath, A. Lozano, T. L. marzetta, and P. Popovski, "Five disruptive technology directions for 5G," IEEE Communications Magazine, vol. 52, pp. 7480, February 2014.

5. T. S. Rappaport et al., "Millimeter wave mobile communications for 5G cellular: It will work!" IEEE Access, vol. 1, pp. 335-349, May 2013.

6. O. E. Ayach, S. Rajagopal, S. Abu-Surra, Z. Pi, and R. W. Heath, "Spatially sparse precoding in millimeter wave MIMO systems," IEEE Trans. on Wireless Communications, vol. 13, pp. 1499-1513, March 2014.

7. A. Alkhateeb, O. E. Ayach, G. Leus, and R. W. Heath, "Channel estimation and hybrid precoding for millimeter wave cellular systems," IEEE J. Select. Topics Signal Process, vol. 8, pp. 831-846, July 2014.

8. R. W. Heath, N. Gonzalez-Prelcic, S. Rangan, W. Roh, and A. M. Sayeed, "An overview of signal processing techniques for millimeter wave MIMO systems," IEEE J. Select. Topics Signal Process, vol. 10, no. 3, pp. 436453, April 2016.
9. J. Mo and R. W. Heath, "Capacity analysis of one-bit quantized MIMO systems with transmitter channel state information," IEEE Trans. on Signal Processing, vol. 63, pp. 5498-5512, July 2015.

10. R. Roy and T. Kailath, "ESPRIT-estimation of signal parameters via rotational invariance techniques," IEEE Trans. on Acoustic, Speech, and Signal Processing, vol. 37, pp. 984-995, July 1989.

11. M. C. Vanderveen, C. Papadias, and A. Paulraj, "Joint angle and delay estimation (JADE) for multipath signals arriving at an antenna array," IEEE Communications Letters, vol. 1, pp. 12-14, January 1997.

12. A. van der Veen, M. C. Vanderveen, and A. Paulraj, "Joint angle and delay estimation using shift-invariance techniques," IEEE Trans. on Signal Processing, vol. 46, pp. 405-418, February 1998.

13. Chahbi and B. Jouaber, "A joint AOA, AOD and delays estimation of multipath signals based on beamforming techniques," IEEE Conference on Signals, Systems and Computers (ASILOMAR), pp. 603-607, November 2010.

14. Lee, G.-T. Gil, and Y. H. Lee, "Channel estimation via orthogonal matching pursuit for hybrid MIMO systems in millimeter wave communications," IEEE Trans. on Communications, vol. 64, pp. 2370-2386, April 2016.

15. S. Rangan, T. S. Rappaport, and E. Erkip, "MillimeterWave Cellular Wireless Networks: Potentials and Challenges," Proceedings of the IEEE, vol. 102, no. 3, pp. 366-385, March 2014

16. M. R. Akdeniz et al., "Millimeter wave channel modeling and cellular capacity evaluation," IEEE Journal on Selected Areas in Communication, vol. 32, pp. 11641179, June 2014.

17. W.-R. Wu, R.-C. Chiueh, and F.-S. Tseng, "Channel estimation for OFDM systems with subspace persuit algorithm," Green Circuits and Systems (ICGCS), pp. 269-272, 2010.

18. G. J. McLachlan and T. Krishnan, The EM Algorithm and Extensions, D. Balding et al., Eds. 111 River Street, Hoboken, NJ 07030: John Wiley \& Sons, Inc., 1997.

19. T. K. Moon, "The expectation-maximization algorithm," IEEE Signal Processing Magazine, vol. 13, pp. 47-60, November 1996.

20. C.-Y. Chen, W.-R. Wu, and C.-S. Gau, "Joint AoA and Channel Estimation for SIMO- OFDM Systems: A Compressive-Sensing Approach," Journal of Signal Processing Systems, vol. 83, pp. 191-205, May 2016. 